This is an electronic reprint of the original article. This reprint may differ from the original in pagination and typographic detail.

Author(s): Jiang, Fan; Käkölä, Timo

Title: $\quad$ Requirements for using simulation to design and assess wireless ATM handover solutions

Year: $\quad 1999$

Version:

Please cite the original version:

Jiang, F.; Käkölä, T.; , "Requirements for using simulation to design and assess wireless ATM handover solutions," Wireless Communications and Networking Conference, 1999. WCNC. 1999 IEEE , vol., no., pp.1008-1012 vol.2, 1999. doi: 10.1109/WCNC.1999.796823

All material supplied via JYX is protected by copyright and other intellectual property rights, and duplication or sale of all or part of any of the repository collections is not permitted, except that material may be duplicated by you for your research use or educational purposes in electronic or print form. You must obtain permission for any other use. Electronic or print copies may not be offered, whether for sale or otherwise to anyone who is not an authorised user. 


\section{Requirements for Using Simulation to Design and Assess Wireless ATM Handover Solutions}

\author{
Fan Jiang \\ Nokia Telecommunications Oy \\ Switching Platforms \\ Torikatu 6, FIN-44100, Äänekoski, Finland \\ fan.jiang@ntc.nokia.com
}

\author{
Timo Käkölä \\ University of Jyväskylä \\ Computer Science and Information Systems \\ FIN-40351 Jyväskylä, Finland \\ timo.kakola@cc.jyu.fi
}

\begin{abstract}
Wireless ATM (WATM) has been widely considered as a solution for broadband wireless services. Handover is one of the most important mobility support functions in the WATM network. Many handover solutions have been proposed, analysed, and benchmarked. Simulations have been broadly used to assess these solutions. However, there are many dilemmas in how simulation has been applied as a research methodology: heterogeneous simulation systems used, lack of comparison with other related work, biased conclusions, and unavailability of simulation models and systems to researchers. The WATM research community lacks a standard and commonly acceptable methodology for applying simulation. To address this problem, a framework for using simulation in assessing wireless ATM handover solutions is proposed. The framework is applied and analysed by implementing a handover simulation model with the COMNET III ${ }^{\mathrm{TM}}$ simulation package.
\end{abstract}

\section{INTRODUCTION}

Wireless ATM (WATM) [1] has been widely considered as a solution for broadband wireless services. A wireless ATM network is an integration of an access network with wireless access points (AP) and a 'mobile ATM' backbone which supports mobility functions.

The mobility functions are (1) location management which resolves the mobile user's location and (2) handover control which maintains the mobile user's call connectivity when it moves from one wireless access point to another [3]. In recent years, both researchers [1] [2] [3] [4] [5] [6] [7] [8] [9] [10] [11] [12] [13] and standardisation bodies such as the ATM Forum [15] [16] have investigated handover control solutions. Many handover solutions have been proposed, analysed and benchmarked. They differ in the alternative methods of handover initiation, path re-routing, and crossover switch (COS) discovery. All solutions are designed to meet specific performance criteria such as complexity, delay, latency, bandwidth, network resource consumption, and efficiency.

Simulation is broadly used as a research methodology to assess the proposed handover mechanisms. This paper reviews different simulations for the wireless ATM handover mechanism. The problem addressed in this paper is that the WATM research community lacks a standard and commonly acceptable methodology for applying simulation. To address this problem, a framework for using simulation in assessing wireless ATM handover solutions is proposed. The framework is applied and analysed when implementing a handover simulation model with COMNET $\Pi^{\mathrm{TM}}$ simulation package.

In section II, the simulation model architecture for wireless ATM handover is explained. The reasons why simulation is a viable research methodology are described. In section III, dilemmas in applying simulation to assess wireless ATM handover solutions are identified. In section IV, the framework is presented. In section V, COMNET IIIM is used to evaluate the framework by building a model for WATM handover. In section VI, we conclude that the framework serves as a useful guideline for simulation package selection and model building.

\section{SIMULATION IN WIRELESS ATM HANDOVER SOLUTIONS}

\section{A. Why Simulation}

Big projects like RACE and COBRA can build real network systems to test handover algorithms or protocols at a small range. But for most researchers it is extremely expensive and impractical. There is not enough resources and time available. Simulation provides an economic and effective way for developing and validating the theory.

Simulation results will not always be in favour of the original theory. When this happens, simulation results serve as feedback for the theory. The theory can thus be improved. This is an enhancement cycle.

Statistical analysis of the simulation results can show convincingly how much a new protocol increases efficiency compared to earlier ones. A research paper with a new mechanism proposed but no simulation implemented will thus stand less chance to be accepted by conference committees or joumal editors. 


\section{B. Basic Simulation Model Architecture}

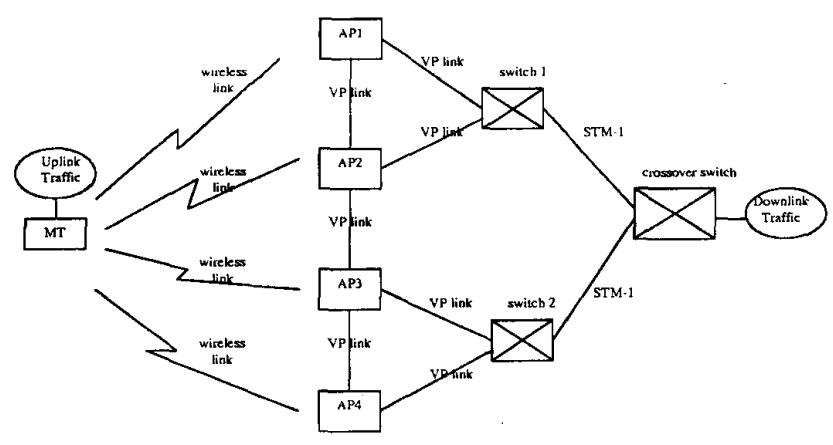

Fig. 1. Simulation model architecture for WATM handover

The basic simulation model architecture for wireless ATM handover is shown in Figure 1. This architecture supports both inter-switch and intra-switch handover mechanisms. Suppose the Mobile Terminal (MT) is originally connected to AP1. When MT moves, the call could be handed over to AP2 as an intra-switch handover or to AP3 or AP4 as an inter-switch handover.

\section{DILEMMAS IN APPLYING SIMULATION IN WATM HANDOVER RESEARCH}

We reviewed WATM research literature to understand how simulation has been applied in analysing handover solutions. Sometimes we got confused about which simulation software package was used, where to find it, what kind of coding was done, where did the results come from, and how to compare the proposed mechanism to others? We started to look further and found the following problems concerned with simulation.

\section{Heterogeneous simulation systems used}

At least the following simulation systems have been used in WATM research: the mobile communications system model (based on BONeS software) [6], Ptolemy [7], simulation package SIMAN/UNIX [13], and CLASS [11]. In some other papers, simulations were mentioned but not described in detail. We started to wonder what kind of a simulation package should be used for assessing handover solutions? What are the criteria for choosing a suitable simulation package?

Lack of comparison with other related work

We noticed that most of the simulations are done not only by different simulators based on different simulation tools, but also under different restrictions and assumptions brought up by authors. A problem occurs when we need to compare among different handover solutions. Because the WATM research community lacks an overall framework for applying simulation methodology, each solution is evaluated using a simulation methodology, which differs from those used in assessing other solutions. It is thus hard to justify the contribution of one
WATM handover solution in relation to other peer solutions and to the effectiveness of the WATM network as a whole. Biased conclusions

Authors typically try to show the solution they proposed is better than others. Thus the results can be biased from the WATM network point of view. If simulations are described and conducted in a way that does not make it easy for other researchers in the WATM field to replicate the simulation studies, how can the accumulation of knowledge be secured?

Unavailable to the public

Designing and programming a simulator tailored only for the specific application may sometimes be viable if the simulation needs are highly specialised. But it is a waste of resources. It also may hamper other researchers from repeating the studies because the simulators are not always made publicly available. If they are made available, they typically are not robust and usable enough so that others could use them easily. Commercial simulation packages solve many of these problems but they are usually expensive and may not be able to simulate specific aspects of WATM networks.

\section{A FRAMEWORK FOR WIRELESS ATM HANDOVER SIMULATION}

Facing the dilemmas described above, we argue that it is indispensable to develop a commonly available and acceptable simulation framework. This could greatly accelerate knowledge creation in the field by eliminating routine work and providing a basis for benchmarking different handover solutions.

In this section we outline such a framework. We discuss generic simulation design principles and then elaborate on architectural, functional, and model component based requirements for a WATM handover simulation system. We have drawn these requirements by (1) reviewing the literature [7][9-14][20] and (2) evaluating a commercial simulation software package COMNET III TM [17][18][19].

These requirements should be revised regularly so that they met new technical developments in the field. The ATM forum would probably be the best organisation to take care of this. The ATM forum might also be able to collaborate with simulation tool vendors so that the tools would evolve together with the requirements. When the standards for using simulation were accepted by the ATM forum, conferences and journals in the field could enforce their use.

\section{A. Simulation Model Design Principles}

In our research we have built a wireless ATM handover model. According to our experiences, the following design principles facilitate the design efforts.

Modelling as an iterative process

Over the course of a simulation study, the model will be refined many times. The end result may look different than the 
original one. The scope of a project often changes which requires the model to change. The modelling process then becomes an iterative process [17].

Seeing the big picture

It is important not to get stuck in every event that occurs on every application at each node in the model. Is it easy to mistakenly try to emulate the network as a whole instead of simulating the model as originally planned. The primary focus should be on capturing the conditions and facts that can have a bearing on the objectives of a simulation. Increase the detail only when necessary [20].

Set simulation goal

Modelling is a way to achieve a goal, not the goal itself. Attainable simulation goals must be set clearly by answering at least the following questions. What do you want to model? What are the assumptions? What are the parameters under study? What can be concluded from the results of the simulation? Only the events that are relevant to the goal are modelled in detail.

Decide modelling strategy

In terms of a simulation strategy, there are two approaches: discrete event simulation (DES) and continuous event simulation. In WATM handover, the most suitable way is DES. In DES, events can be observed as they happen over time and performance measures can be collected. Typical performance measures include capacity utilisation levels, waiting times, and queue sizes.

\section{B. Architectural Requirements for the WATM Handover Simulation System.}

The simulation system architecture has to meet at least the following objectives.

Scalability

New requirements, algorithms, and mechanisms are invented every day. The simulation system should be extendable with new features at minimum effort. Scalability is essential.

Unified and commonly acceptable

The architecture should provide a standardised measurement scheme for benchmarking solutions. It also needs to be acceptable to as wide audience as possible so that critical mass can be achieved to support continuous system development. Ease of use

When the architecture is easy to use, researchers save a lot of time in learning and using it.

Robustness

A robust architecture can be used to assess different handover solutions under as many different circumstances and by as many users as possible.

Versatility and reusability

The architecture should not be limited to Wireless ATM handover, that is, horizontal (peèr) comparison. It should also be applicable for current mobile handover, that is, vertical (different generation) comparison. Versatility facilitates, for example, reuse of current model components in $R \& D$ that have been proven to work in practise. Reusability is also enhanced if the architecture can model call admission control (CAC) because many characteristics of CAC are similar to handover (e.g., signalling, resource allocation, and routing).

\section{Functional Requirements for the WATM Handover Simulation System}

The measurement criteria to be used in WATM simulation include bandwidth, complexity, delay, latency, buffer, and network resource consumption [2]. The simulation system has to meet at least the following requirements.

1) Support the following types of handover from the viewpoint of network handover (inter-switch handover, intraswitch handover): backward handover, soft handover, forward handover, hard handover, MT-initiated handover, and networkinitiated handover.

2) Support different traffic classes (CBR, VBR, UBR and ABR).

3) Support handover latency simulations to test the lossless and seamless handover. Delay-sensitive and delay-insensitive traffic cast different demands on handover duration.

4) Support heterogeneous handover, that is, both unicast and multicast calls.

5) Enable MT to handover to different access points. It should be possible for MT to list more than one candidate AP in a handover request with the assumption that the network then chooses the most appropriate one to use [2].

6) Support exclusive handover. For a multimedia call, more than one connection (audio, video, and data) exists at a given time. All the connections for the WATM call should be handed over simultaneously.

7) Support process intelligence of AP and MT.

8) Support different Quality of Service (QOS) traffic.

9) Support signalling traffic.

10) Support forwarding and buffering because these features are crucial to guarantee lossless handover.

\section{Simulation Components Modelling}

In this section we draw on the simulation model architecture (Figure 1) to discuss the modelling of simulation components. Specify parameters

Parameters to be specified in a WATM handover simulation model include traffic model, buffer, connection link, delay, bandwidth consumption, and cell routing.

Traffic modelling

Traffic modelling is a key issue in network analysis. Traffic in the wireless ATM handover model includes: (1) downstream and upstream traffic load for the particular call which needs handover; (2) background traffic to simulate the real system. Background traffic can be generated using traffic sources or by defining link utilisation. In ATM backbone network, traffic contract need to be agreed including (1) traffic descriptors and 
rules for a compliant connection, (2) QoS parameters, and (3) conformance checking rules. Source traffic descriptors are those parameters negotiated between the user and the network at the time the connection is established. Descriptors PCR, SCR, MBS, and MCR need to be specified [19]. Traffic is divided into delay-sensitive and delay-insensitive traffic. QoS categories (CBR, rt-VBR, nrt-VBR, ABR, and UBR) are chosen to reflect different traffic types. Traffic shaping is usually done by Generic Cell Rate Algorithm (GCRA), also known as the leaky-bucket algorithm [18]. Traditionally, telecommunication traffic scheduling has been modelled using Poisson processes. However, alternatives like Markov and Pareto are also available [13]. A Poisson process generating independent arrivals with exponentially distributed interarrival times is recommended in COMNET III ${ }^{\mathrm{TM}}$ for traffic modelling. Buffer modelling

Research focused on buffer utilisation [7] [11] identified that large buffers should be used to secure a lossless handover for delay-insensitive traffic like file transfers. For each call (there may be more than one connection included) three separate buffers are needed for upstream traffic, downstream traffic and forwarded traffic. Forwarded traffic buffer should be emptied first to maintain the cell sequence of the traffic. Buffers can be in a switch, AP, and MT. The most appropriate place to buffer traffic is AP. This can reduce the complexity of the switch. MT has limited buffer capacity due to its small physical size.

\section{Connection link modelling}

Connection link types are: (1) radio links connecting MT to APs; (2) VP links connecting APs to each other and to the local switch; and (3) fibre optic links connecting the local switch to the cross-over switch. Each link type has its own characteristics such as the data rate that needs to be specified.

Modelling Delay

There are two kinds of delay: propagation delay for each link type and process delay on each node (MT, AP, and switch). Modelling bandwidth consumption

When MT starts a call, radio bandwidth will be allocated for upstream traffic as well as downstream traffic. Bandwidth on each link of the route will also be allocated to route the call.

\section{Modelling packet routing}

Applying different routing protocols can result in different connection routes during the handover. Connection-oriented routing is used to model ATM connections. The routing protocols built into COMNET IIITM are IGRP; Link-State Shortest-Path First; RIP Minimum Hop; Minimum Penalty; Shortest Delay and User-Defined Routing Tables. In UserDefined Routing Tables, where a user enters a number of possible routes, the selection criteria are: First Available; Max Unused Bandwidth; Min Delay; Min Queue; Min Sessions; Random List; and Round Robin.

\section{E. Process Simulation Statistics}

Simulation statistics are analysed and compared to assess the original theory. WATM handover solutions pose no particular statistical requirements, which most commercial tools could not handle.

\section{APPLYING THE FRAMEWCRK TO EVALUATE COMNET III ${ }^{\mathrm{TM}}$}

We have built an intra-switch handover model on COMNET $\mathrm{II}^{\mathrm{TM}}$ to apply and assess the simulation framework. We used parameters stated in [7][11][12][13][14] to test the scalability of the simulation system. Figure 2 shows the model.

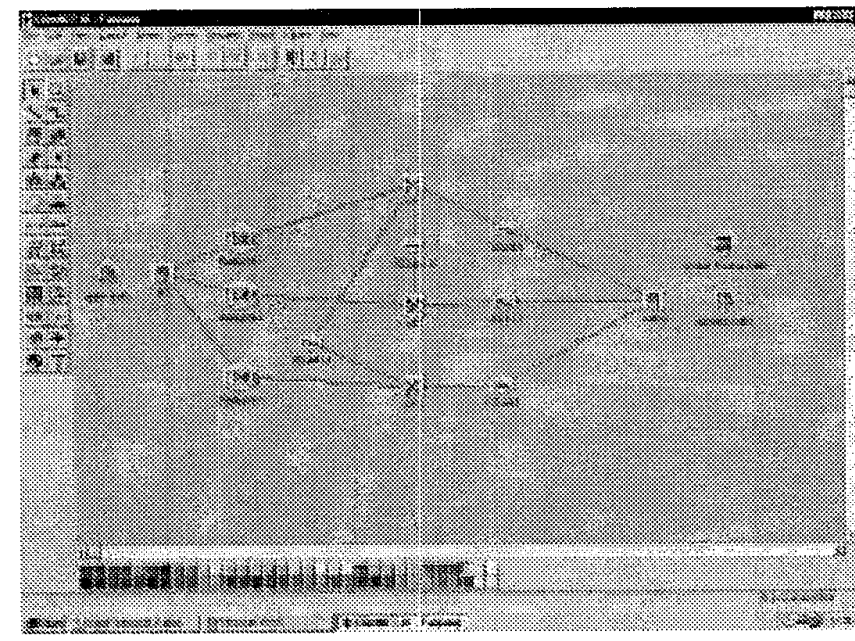

Fig. 2: Intra-swịtch handover model

Through modelling, COMINET III'M proved to meet most simulation requirements. The graphical user interface and ready-made libraries make it easy to use. User chooses the components (e.g., node, link) and defines the parameters for them. No coding is needed. ATM Forum standard is applied for ATM traffic. This helps make the system unified and commonly acceptable. Other types of mobile handover (e.g., satellite mobile) can also be modelled. However, limitations exist. (1) In traffic modelling, alternatives like Markov and Pareto are missing. This can reduce the scalability of the system. (2) Nodes used to model MT, AP, and switch can not allocate buffer at wish for forward traffic. This hinders the buffer modelling for lossless handover. (3) Application source does not have enough intelligence to model the signalling traffic. (4) Exclusive handover: is not supported.

Commercial telecommunication simulation software packages such as COMNET IUIM can not be targeted solely for a particular application. On the other hand, new algorithms and mechanisms are invented frequently, casting new demands on simulation models. It is thus not possible for a simulation package to satisfy all the needs. A wise compromise is to provide application programming interfaces so users can enhance the off-the-shelf package with additional features for special applications. Users thus have more freedom to build models for particular purposes. 


\section{CONCLUSIONS AND FUTURE RESEARCH}

The problem addressed in this paper was that the WATM research community lacks a standard and commonly acceptable methodology for using simulation to assess proposed systems, protocols, and mechanisms. As a result, the accumulation of knowledge in the field is hampered. To address this problem, we proposed a framework for using simulation in WATM handover research. The framework has a dual purpose: (1) Helping WATM researchers conduct simulation studies in a rigorous and common way that facilitates the assessment and benchmarking of proposed solutions; (2) Helping the simulation software industry understand the requirements WATM pose to the software. The framework consists of simulation design principles and architectural, functional, and model component level requirements. A commercial simulation package COMNET III ${ }^{\mathrm{TM}}$ was evaluated according to the simulation framework. It proved to satisfy most of the requirements like scalability, ease of use, and robustness. However, there are requirements COMNET III ${ }^{\mathrm{TM}}$ can not meet such as multimedia call and exclusive handover. Programming interfaces need to be incorporated into off-the-shelf packages so researchers can implement new features as necessary.

This paper has focused on handover in the context of smallscale model building. To improve the applicability and usefulness of the framework, future research must extend it to cover related areas such as call admission control. Large WATM projects using multiple simulation platforms must also be conducted and carefully reflected on to further develop the framework.

\section{REFERENCES}

[1] F. Jiang and T. Käkolä, "Wireless ATM: Handover Issues," in the proceedings of PIMRC'98: The Ninth IEEE International Symposium on Personal, Indoor, and Mobile Radio Communications, Boston, Massachusetts, U.S.A., September 8-11, 1998.

[2] F. Jiang and T. Käkölä, "Microcellular Handover in Wireless ATM," in the proceedings of 32nd Annual Hawaii International Conference on System Sciences (HICSS), Maui, Hawaii, U.S.A., January 5-9, 1999.

[3] J. Li, A. Acharya, and D. Raychaudhuri, "Zero-cell-loss Handoff in Wireless ATM Networks," in the proceedings of 32nd Annual Hawaii International Conference on System Sciences (HICSS), Maui, Hawaii, U.S.A., January 5-9, 1999.

[4] C-K. Toh, Wireless ATM and AD-HOC Networks, Kluwer Academic Publishers, 1997.

[5] A. Acampora and M. Naghshineh, "An Architecture and Methodology for Mobile-Executed Handoff in Cellular ATM networks," IEEE Journal on Selected Areas in Communications, vol. 12, no. 8, pp. 1365-1374, December 1994.
[6] R. Yuan, S.K. Biswas, L. J. French, J. Li, and D. Raychaudhuri, "A Signaling and Control Architecture for Mobility Support in Wireless ATM network," ACM/Baltzer Mobile Networks and Applications, vol. 1, no. 3, pp. 287-298, December 1996.

[7] H. Mitts, H. Hansen, J. Immonen, and S. Veikkolainen, "Lossless Handover from Wireless ATM," ACM/Baltzer Mobile Networks and Applications, vol. 1, no. 3, pp. 299-312, December 1996.

[8] A. Acharya, J. Li, and D. Raychauhuri, "Mobility Management in Wireless ATM networks," IEEE Communication Magazine, vol. 35, no. 11, pp. 100-109, November 1997.

[9] M. Veeraraghavan, M. Karol, and K. Eng, "Mobility and Connection Management in a Wireless ATM LAN," IEEE Journal on Selected Area in Communications, vol. 15 , no. 1, pp. 323-339, January 1997.

[10] C. F. Chiasserini and R. Lo Cigno, "Handovers in Wireless ATM: An In-Band Signalling," in the proceedings of Universal Personal Communications (ICUPC'98), pp. 775-779, 1998.

[11] M. Ajmone Marsan, C. F. Chiasserini, A. Fumagalli, R. Lo Cigno, and M. Munafo, "Buffer Requirements for Loss-Free Handovers in Wireless ATM Networks," IEEE ATM Workshop, pp. 416-424, 1997.

[12] A. G. Kaloxyos, G. Papageorgiou, P. Papageorge, and L. Merakos, "Smart Buffering Technique for Lossless Hard Handover in Wireless ATM Networks," Universal Personal Communications Record, pp. 300-305, 1997.

[13] M. Kang, M. Jung, and S. Lee, "A Stochastic Rerouting Mechanism for Handoff in Mobile ATM Network," in the proceedings of PIMRC'98: The Ninth IEEE International Symposium on Personal, Indoor, and Mobile Radio Communications, vol. 1, pp. 345-349, Boston, Massachusetts, 1998.

[14] E. Gustafsson and R. Rönngren, "Fluid Traffic Modelling in Simulation of a Call Admission Control Scheme for ATM Networks," in the proceedings of Modeling, Analysis, and Simulation of Computer and Telecommunication Systems (MASCOTS'97), pp. 110115, 1997.

[15] The ATM Forum, Draft Wireless ATM Requirements Specification, RTD-WATM-01.03, February 8, 1999.

[16] The ATM Forum, Draft Wireless ATM Capability Set 1 Specification, BTD-WATM-01.10, December 19, 1998.

[17] COMNET III ${ }^{\mathrm{TM}}$, Getting Started Guide, Release 1.4, CACI.

[18] COMNET IIITM, Modelling ATM Networks With COMNET III ${ }^{\mathrm{TM}}$, Version 1.0, CACI.

[19] COMNET III ${ }^{\mathrm{TM}}$, Reference Guide, Release 2.0, CACI.

[20] T.J. Gogg and J.R.A. Mott, "Improve Quality \& Productivity With Simulation," 3rd ed., JMI Consulting Group, chapter 11.4-3, 1996. 